\section{(2) OPEN ACCESS}

\title{
Structuring mental health support for frontline caregivers during COVID-19: lessons from organisational scholarship on unit-aligned support
}

\author{
Julia DiBenigno ๑ , ${ }^{1}$ Michaela Kerrissey $๑^{2}$
}

\begin{abstract}
${ }^{1}$ Organizational Behavior, Yale School of Management, New Haven, Connecticut, USA ${ }^{2}$ Department of Health Policy and Management, Harvard University T H Chan School of Public Health, Boston, Massachusetts, USA
\end{abstract}

Correspondence to Dr Julia DiBenigno, Organizational Behavior, Yale School of Management, New Haven, CT 06511, USA; julia.dibenigno@yale.edu

Received 1 May 2020 Revised 8 May 2020 Accepted 12 May 2020 Published Online First 2 June 2020

\begin{abstract}
Background Although the COVID-19 pandemic exposes frontline caregivers to severe prolonged stresses and trauma, there has been little clarity on how healthcare organisations can structure support to address these mental health needs. This article translates organisational scholarship on professionals working in organisations to elucidate why traditional approaches to supporting employee mental health, which often ask employees to seek assistance from centralised resources that separate mental health personnel from frontline units, may be insufficient under crisis conditions. We identify a critical but often overlooked aspect of employee mental health support: how frontline professionals respond to mental health services. In highrisk, high-pressure fields, frontline professionals may perceive mental health support as coming at the expense of urgent frontline work goals (ie, patient care) and as clashing with their central professional identities (ie, as expert, self-reliant ironmen/women).
\end{abstract}

Findings To address these pervasive goal and identity conflicts in professional organisations, we translate the results of a multiyear research study examining the US Army's efforts to transform its mental health support during the wars in Iraq and Afghanistan. We highlight parallels between providing support to frontline military units and frontline healthcare units during COVID-19 and surface implications for structuring mental health supports during a crisis. We describe how an intentional organisational design used by the US Army that assigned specific mental health personnel to frontline units helped to mitigate professional goal and identity conflicts by creating personalised relationships and contextualising mental health offerings.

Conclusion Addressing frontline caregivers' mental health needs is a vital part of health delivery organisations' response to COVID-19, but without thoughtful organisational design, well-intentioned efforts may fall short. An approach that assigns individual mental health personnel to support specific frontline units may be particularly promising.

\section{INTRODUCTION}

The COVID-19 pandemic has unleashed extraordinary stresses on frontline caregivers, from personal exposure risk and fears of infecting loved ones to extreme and unfamiliar workloads while facing moral dilemmas and intense suffering in patient care. Healthcare organisations' ability to support frontline caregivers' mental health needs is widely considered essential as the pandemic draws ongoing waves of critically ill patients to their doors. ${ }^{1}$
However, the call to care for others often eclipses caregivers' attention to their own well-being, and even under typical circumstances, they suffer from high rates of burnout, stress, trauma and suicide. ${ }^{2}$ These challenges are amplified by the pandemic; for instance, a survey of COVID-19 caregivers in China found that $72 \%$ reported distress; $50 \%$ reported depression; and $45 \%$ reported anxiety. ${ }^{3}$ Healthcare organisations report that traditional approaches to supporting employee mental health, such as employee assistance programmes, are falling short under the present crisis conditions. ${ }^{4}$ Yet, there has been little clarity on why this is the case and what alternatives may be better.

Translating findings from organisational scholarship on professionals working in organisations during rapid change, this paper informs the vital question of how to support professional caregivers' mental health needs by elucidating professionals' reactions to organisationally sponsored mental health services. Organisational scholarship has demonstrated how professionals experience goal and identity conflicts that undermine frontline professional cooperation with organisational support efforts. ${ }^{5}{ }^{6}$ Frontline leaders and workers, especially in fields characterised by high stress and high risk, may perceive mental health supports as distracting from all-consuming frontline work goals (ie, patient care) and as going against their valued professional identities (ie, as expert, self-reliant ironmen/women). Because most traditional organisational mental health structures put the onus on employees to seek help and separate mental health personnel from frontline units, they neglect to create opportunities to develop the familiarity and contextualised awareness between mental health personnel and frontline units that can help to align seemingly conflicting goals and bridge identity differences. Without attention to these critical issues-and intentional efforts to design mental health offerings that address them-well-intended efforts to meet caregivers' mental health needs during COVID-19 are likely to fall short.

This paper proceeds in three parts. First, we synthesise organisational research on professionals in organisations to articulate how goal and identity conflicts arise within organisations and elucidate why these conflicts can undermine well-intended organisational efforts to support professionals during a crisis. Second, we describe findings from a major multiyear ethnographic research study examining an effort to deliver mental health support in a professional arena with strong parallels to healthcare during COVID-19: the US Army during the wars in Iraq and Afghanistan. 
This in-depth translation of a research study in a parallel context illustrates the problems that professional goal and identity conflict can pose for providing crisis-related mental health support and surfaces an alternative approach that is rooted in research and theory on organisational design. Third, we discuss the implications of the Army study for healthcare organisations and highlight the potential for skilful organisational design to help address goal and identity conflicts by assigning mental health personnel to support specific frontline units. These lessons from organisational scholarship and the US Army's experience provide critical insight into how healthcare organisations can structure mental health support for frontline caregivers during COVID-19.

\section{ORGANISATIONAL BARRIERS TO MENTAL HEALTH SUPPORT: GOAL AND IDENTITY CONFLICT IN PROFESSIONAL ORGANISATIONS}

Scholarship on professionals in organisations elucidates two important barriers to the use and effectiveness of employee mental health support during crises: goal conflict and identity differences between frontline units and professional mental health personnel, described in detail as follows.

\section{Goal conflict}

Classic organisational scholarship finds goal conflict is endemic within organisations, as specialised units and different professional groups often have their own interests and prioritise their own goals which may conflict. ${ }^{5-7}$ Even when shared organisational goals exist, such as to support the mental health of an organisation's workforce while ensuring quality service delivery, entrenchment in one's own group's perspective from their professional training and position in the organisational structure can make shared goals difficult to achieve. This can lead to regular conflict between frontline units and members of professional groups brought in to support them, leading to suboptimal outcomes. ${ }^{589}$

When implementing mental health support in frontline healthcare environments, perceptions of goal conflict are reasonable to expect. Both frontline unit leaders and staff may view mental health support as detracting from patient care goals; for example, research in surgery finds perceptions of a zero-sum conflict between being 'ironmen' who are fully dedicated to patient care 24/7 and complying with wellness-oriented interventions that limit work hours to promote sleep and prevent burnout. ${ }^{10}$ Such goal conflicts are likely further heightened in a crisis that accentuates time constraints, as COVID-19 has done. Taking time away from care delivery for mental health may be seen as creating further team burdens on the unit, a common saying within healthcare being 'if you are not rounding, you are being rounded on', implying the only acceptable excuse for not providing patient care is becoming a patient oneself. By contrast, in line with their own professional training, mental health personnel may prioritise the mental health needs of caregivers over frontline units' near-term patient care goals.

Organisational research has identified a number of mechanisms through which goal conflict can be addressed. These include, for example, establishing formal rules and guidelines for interaction across groups ${ }^{7}$; establishing cross-functional teams, task forces and departments ${ }^{11}$; implementing collaborative incentives ${ }^{12}$; and deploying colocation or matrix structures. ${ }^{13} 14$ These mechanisms rationally rely on the idea that better aligning goals through rules, incentives and authority structures will be sufficient to bridge goal conflicts; however, many conflicts in organisations have proven immune to such rational attempts at goal alignment. ${ }^{5615}$ When there are deeply held differences in identities between groups-as there likely are between those on frontline units and mental health support personnel-such rational mechanisms can prove ineffective.

\section{Identity conflict}

Identity differences between professional groups and departmental units within organisations can exacerbate goal conflicts, making them heated and personal. Identity refers to how a group collectively defines 'who they are', including their distinctive values, beliefs and sense of what being a good-standing member entails, and they are often apparent in members' common dress, language and demeanour. ${ }^{16}$ Identity differences are prevalent across professional groups, for whom 'who they are' (their professional identity) is intimately connected with 'what they do' as professionals. ${ }^{17}$ Professional group members are often especially committed to advancing goals congruent with their strongly held professional identities. ${ }^{18}$ For example, in many healthcare specialties, training and culture prize professional identities in which one is tough, desensitised and self-reliant in response to traumatic situations. These qualities are largely considered part of being a good professional caregiver, what has been described as the 'historic "iron doc" culture' of medicine. ${ }^{19}$ Such professional identities run counter to identities associated with mental health professionals as supporting vulnerability and seeking help. The prevalent labeling of frontline caregivers as 'heroes' as they serve amid COVID-19 may further buttress this identity, perhaps making it even harder to overcome the stigma of admitting a need for support and to relate positively with mental health personnel.

Because identity runs deep in individuals, goal conflicts arising across groups with different identities can be difficult to ameliorate through rational means alone. For example, research has documented how inviting physician and hospital administrator groups prioritising different goals (eg, providing quality patient care and managing a profitable hospital) to a strategic planning retreat without addressing identity differences can backfire and further fuel their conflict. ${ }^{20}$ Similarly, because there are likely identity differences between mental health personnel and frontline caregivers, particularly those in emergency and intensive care units known for their stoicism amid trauma, there is potential that mental health resources go underused and underappreciated because they are considered out of touch with the realities of frontline units' unique professional identities.

In sum, organisational scholarship on professionals in organisations suggests goal conflict and identity differences between mental health and frontline caregiver professional groups may pose substantial barriers to the use and usefulness of mental health support during COVID-19.

\section{ALIGNING MENTAL HEALTH SUPPORT WITH FRONTLINE UNITS: THE US ARMY AND ANCHORED PERSONALISATION}

With goal and identity conflict posing stark challenges to providing mental health services to frontline professionals, integrative solutions that equip organisations with practical strategies to address conflicting goals and identity differences are vital. To shed light on potentially useful strategies for structuring mental health support during COVID-19, we further describe findings from a major multiyear ethnographic research study conducted from 2012 to 2015 examining an effort to deliver mental health support in a professional arena with strong parallels to healthcare during COVID-19: the US Army during the wars in Iraq and Afghanistan. ${ }^{21-25}$ 


\section{US Army case: anchored personalisation}

During the wars in Iraq and Afghanistan, the US Army sought to transform its mental health services to better support soldiers' needs, given increased rates of post-traumatic stress disorder and suicide. However, much like healthcare workers during COVID-19, soldiers in the US Army faced the dual challenge of prolonged periods of high personal risk and stress, combined with intense demands to be 'field ready' and 'tough. In-depth research examining 18 Army brigades and mental health clinics over time (through over 300 in-depth interviews and 600 hours of on-site observation) indicated that most traditional mental health support initially went underused, due to many of the professional goal and identity issues described earlier. Mental health support provoked resistance from soldiers' supervisory commanders, who often discouraged soldiers from using mental health services, fought treatment recommendations (eg, to allow a soldier to sit out of a stressful training exercise) and emphasised long-standing prejudices about mental health personnel (eg, calling them 'Berkeley hippies'). Meanwhile, mental health personnel, stereotyping commanders as 'bullies', remained removed from soldiers' unique units and work environments, and made recommendations often considered inappropriate for valued mission-readiness goals or unnecessarily damaging to soldier career aspirations.

However, this research also uncovered an alternative approach that resolved the pervasive and long-standing goal and identity conflicts between mental health support and frontline unit supervisors and members. Rather than providing centralised resources that soldiers were expected to proactively seek out, a dedicated mental health clinician was assigned to work specifically with a few frontline units. This was the structure ultimately implemented across the US Army after experimenting with numerous other structures. ${ }^{21} 22-25$ This approach enabled what is called 'anchored personalisation'. ${ }^{21}$

'Personalisation' occurred as mental health personnel developed personalised relationships and familiarity with the frontline unit members and leaders they were assigned to support. This both helped mental health personnel to customise the support they offered to suit the unique needs of individuals in their specific units and to reduce the stigma supervisory commanders and soldiers attached to mental health services that ran counter to their selfreliant 'warrior' identities. In working with and learning about specific units, mental health personnel were able to design support that was sensitive to the specific mental health needs of soldiers in their units, as well as their career aspirations and unit goals (eg, discretely assigning a soldier to a less stressful role during a training exercise). Because these mental health personnel saw patientsoldiers in the same units, they also learned about unit-level issues that helped them tailor supports. Making mental health personnel accountable for learning about the unique needs of their assigned units spurred them to devise support that resonated with rather than conflicted with frontline professional identities. In so doing, feelings of stigma began to change; for example, using mental health services started being framed as 'a sign of strength' or 'being man enough to get help', which aligned with the professional identities of many in all-male combat units. At the same time, this approach ensured this personalisation was balanced by 'anchoring', in which the mental health personnel who were assigned to different units regularly came together, helping one another remain anchored in their professional goals of supporting mental health and resist the demands of frontline units' leadership that sometimes ran counter to soldier well-being.
The concept of anchored personalisation for mental health support provision is rooted in organisational scholarship on personalisation. Research on personalisation, defined as regular, individuated one-on-one contact across groups, has been found to reduce intergroup stereotyping and lead to increased perspectivetaking as members of different groups develop familiarity with and knowledge about one another as people rather than stereotypes in a variety of contexts. ${ }^{26}$ Such perspective-taking can enable people to break out of their entrenched worldviews to find integrative solutions that are win-win for both groups. However, personalisation can also become problematic if personalised contact with the other group leads to co-optation and indoctrination into the other group's perspective, a phenomenon exhibited among bankers through 'regulatory capture ${ }^{27}$ and affirmative action officers protecting their organisations over advocating for employees. ${ }^{28}$ It is for this reason that maintaining an anchoring contact with one's home group is vital for mitigating risks of co-optation when personalisation transpires. ${ }^{21}$

\section{STRUCTURING MENTAL HEALTH SUPPORT AMID COVID-19}

Although there are certainly differences between professional soldiers who train to endure prolonged traumatic situations at war and frontline caregivers suddenly confronting unexpected traumatic situations brought by the COVID-19 pandemic, there are two central insights from the US Army's experience structuring mental health support that are especially relevant for the challenge of providing mental health support for frontline caregivers. First, if personalisation is vital to breaking down barriers to mental health across professional differences, then relying on it to happen by chance is likely insufficient, particularly in high-risk, high-pressure fields like the military and healthcare. Because organisational structures shape the types of interactions members have with others inside their organisations, when properly designed, the right structures can offer opportunities for regular interaction, familiarity and personal relationships between members of the organisation outside one's home group. ${ }^{29}$ In healthcare, organisational design is a critical lever for structuring and institutionalising effective care delivery. ${ }^{30}$ Intentional organisational design may be a key lever through which healthcare organisations can ensure mental health offerings achieve their intended impact.

The second central insight emerging from the US Army case is that the specific strategy of assigning mental health personnel to a few specific frontline units may be particularly advantageous. Because it enables anchored personalisation, this strategy may help mitigate goal conflict and identity challenges related to mental health support usage in healthcare, such as limited time and a culture of 'toughing it out', that make mental health resources so difficult for caregivers to seek out and use in practice. If unit staff, and particularly unit leaders, become more familiar with a dedicated mental health support person through personalised interactions, together they can break down stigma and stereotypes, take one another's perspectives and become partners in jointly devising contextualised ways of supporting unit staff well-being that are customised and minimally disruptive to patient care. Doing so may also help personnel frame mental health services in ways that resonate with specific units to encourage use, such as by relabeling mental health as 'psychosocial support' or 'resiliency coaching', or by tapping into the self-sacrificing identity of many caregivers through emphasising how use of mental health support may enable sustained high quality care for patients. ${ }^{31}$

Customisation of mental health support is particularly critical because the specific stresses brought on by COVID-19 vary across 
different departments and units. For example, the mental health needs of a COVID-19 intensive care unit with nurses isolated behind closed doors with gravely ill patients may differ from those of emergency department staff interacting with large numbers of patients whose COVID-19 status may yet be unknown. In addition, time constraints across units vary; for example, especially busy units may require more proactive identification of who needs help, such as from assigned peer 'buddies' or leaders, while others may require daily decompression huddles with their assigned mental health personnel purposefully making themselves available afterward to do one-on-one follow-ups. At the same time, ensuring mental health personnel maintain connection with those in similar roles serving other units can help them stand firm on the importance of supporting mental health goals, even when time demands and the culture of medicine may default to relegating mental health back to the sidelines.

\section{CONCLUSION}

Addressing caregivers' mental health needs is a vital part of health delivery organisations' response to the COVID-19 crisis, but without thoughtful organisational design, even wellintentioned efforts may fail. Organisational research elucidates how goal and identity conflict can undermine such efforts in professional organisations and has explored possible solutions that can be built into the design of mental health support. Based on findings from a in-depth study of the US Army's evolution of its mental healthcare support for frontline units, a design that assigns individual mental health personnel to support specific frontline units may be particularly promising during COVID-19. Because it enables anchored personalisation, this design can help professional caregivers and mental health personnel bridge their differences and devise innovative solutions that increase the use and usefulness of mental health support. Implementation of versions of anchored personalisation in response to COVID-19 is already occurring, with examples in systems such as University of California's Zuckerberg San Francisco General Hospital and Yale New Haven Hospital. These and related efforts to thoughtfully apply organisational structures to better support caregiver mental health will remain vital as the early crisis fades, but the pressure on frontline caregivers endures and resurfaces as the pandemic continues. Perhaps such efforts will also help revitalise how mental health, stress, trauma exposure and burnout are addressed in healthcare delivery, even after this crisis, to better support the frontline caregivers who serve patients day after day.

\footnotetext{
Acknowledgements We are grateful to the healthcare leaders and frontline caregivers working during the COVID-19 pandemic for whom we hope this article will be of service to. We also thank Amy Wrzesniewski and Marissa King for reading a prior version, Amit Nigam for his encouragement to write this translation piece, and the BMJ Leader editorial team for thier rapid turnaround of this work. The views and conclusions contained herein are those of the authors and do not necessarily reflect the views of the US government or US Army.
}

Contributors JDiB and MK played an equal role in conceptualising and writing this article.

Funding The authors have not declared a specific grant for this research from any funding agency in the public, commercial or not-for-profit sectors.

Competing interests None declared.

Patient consent for publication Not required.

Provenance and peer review Not commissioned; externally peer reviewed.

Open access This is an open access article distributed in accordance with the Creative Commons Attribution Non Commercial (CC BY-NC 4.0) license, which permits others to distribute, remix, adapt, build upon this work non-commercially, and license their derivative works on different terms, provided the original work is properly cited, appropriate credit is given, any changes made indicated, and the use is non-commercial. See: http://creativecommons.org/licenses/by-nc/4.0/.

\section{ORCID iDs}

Julia DiBenigno http://orcid.org/0000-0001-6975-110X

Michaela Kerrissey http://orcid.org/0000-0002-4366-1322

\section{REFERENCES}

1 Shanafelt T, Ripp J, Trockel M. Understanding and addressing sources of anxiety among health care professionals during the COVID-19 pandemic. JAMA 2020. doi:10.1001/jama.2020.5893. [Epub ahead of print: 07 Apr 2020].

2 Ruotsalainen JH, Verbeek JH, Mariné A, et al. Preventing occupational stress in healthcare workers. Cochrane Database Syst Rev 2014;11.

3 Lai J, Ma S, Wang Y, et al. Factors associated with mental health outcomes among health care workers exposed to coronavirus disease 2019. JAMA Netw Open 2020;3:e203976

$4 \mathrm{Kim}$ S, Kritek P, Lynch JB, et al. All hands on deck: how UW medicine is helping its staff weather a pandemic. NEJM Catal 2020

5 Cyert RM, March JG. A behavioral theory of the firm. Englewood cliffs. NJ: PrenticeHall, 1963.

6 Fiol CM, Pratt MG, O'Connor EJ. Managing intractable identity conflicts. AMR 2009;34:32-55.

7 March JG, Simon HA. Organizations. New York, NY: John Wiley \& Sons, 1958.

8 Nigam A. Multiple and competing goals in organisations: insights for medical leaders. BMJ Leader 2018;2:85-6.

9 Nigam A, Huising R, Golden B. Explaining the selection of Routines for change during organizational search. Adm Sci Q 2016;61:551-83.

10 Kellogg KC. Challenging operations: medical reform and resistance in surgery. University of Chicago Press, 2011.

11 Pinto MB, Pinto JK, Prescott JE. Antecedents and consequences of project team crossfunctional cooperation. Manage Sci 1993;39:1281-97.

12 Kretschmer T, Puranam P. Integration through incentives within differentiated organizations. Organization Science 2008:19:860-75.

13 Lawrence PR, Lorsch JW. Differentiation and integration in complex organizations. Adm Sci Q 1967a;12:1-47.

14 Mintzberg H. Structure in Fives: designing effective organizations. Englewood Cliffs, NJ: Prentice-Hall, 1993.

15 Jarzabkowski P, Balogun J. The practice and process of delivering integration through strategic planning. J Manag Stud 2009;46:1255-88.

16 Bartel CA, Blader S, Wrzesniewski A. Identity and the modern organization. New York, NY: Psychology Press, 2012.

17 DiBenigno JM, Kellogg KC. Beyond occupational differences: the importance of crosscutting demographics and dyadic toolkits for collaboration in a U.S. Hospital. Adm Sci Q 2014:59:375-408.

18 Anteby M, Chan C, DiBenigno JM. Three lenses on professions and occupations in organizations: becoming, doing, and relating. Acad Manag Ann 2016;10:183-244.

19 Bohman B, Dyrbye L, Sinsky C, et al. Physician well-being: the reciprocity of practice efficiency, culture of wellness, and personal resilience. NEMJ Catal 2017;3.

20 Wiedner R, Nigam A, da Silva J. GP's are from Mars, administrators are from Venus: the role of misaligned occupational dispositions in inhibiting mandated role change. Work Occup 2020.

21 DiBenigno JM. Anchored personalization in managing goal conflict between professional groups: the case of U.S. Army mental health care. Adm Sci Q 2018;65.

22 DiBenigno J. Rapid Relationality: how peripheral experts build a foundation for influence with line managers. Adm Sci Q 2020;65:20-60.

23 Srinivasan J, Carroll J, DiBenigno J, et al. US Army: transformation to a behavioral health system of care.. In: Kochan T, Glasmeier T, Larson T, et al, eds. Post-Traumatic stress innovations final report: transforming the psychological health system of care in the US military. Cambridge, MA: Massachusetts Institute of Technology, 2016.

24 Srinivasan J, DiBenigno J, Carroll J. Transformation of the US Army behavioral health system of care: an organizational analysis using the 'three lenses. Organ Behav J 2017:5-18.

25 Hoge CW, Ivany CG, Brusher EA, et al. Transformation of mental health care for U.S. soldiers and families during the Iraq and Afghanistan wars: where science and politics intersect. Am J Psychiatry 2016;173:334-43.

26 Ensari NK, Miller N. The application of the personalization model in diversity management. Group Processes \& Intergroup Relations 2006;9:589-607.

27 Katic I, Kim JW. Caught in the revolving door: firm-government ties as determinants of regulatory outcomes. Academy of Management Proceedings 2013;2013:12899.

28 Edelman LB, Petterson S, Chambliss E, et al. Legal ambiguity and the politics of compliance: affirmative action officers' dilemma. Law Policy 1991;13:73-97.

29 Blau PM. Structural contexts of opportunities. Chicago, IL: University of Chicago Press, 1994.

30 Valentine MA, Edmondson AC. Team scaffolds: how mesolevel structures enable rolebased coordination in temporary groups. Organization Science 2015;26:405-22.

31 Grant AM, Berry JW. The necessity of others is the mother of invention: intrinsic and prosocial motivations, perspective taking, and creativity. AMJ 2011;54:73-96. 